\title{
Exploring the Diagnosis and Management of Bouveret's Syndrome
}

\author{
Mukul Bhattarai,, ${ }^{1}$ Pardeep Bansal, ${ }^{1}$ Bharat Patel, ${ }^{2}$ Alexander Lalos ${ }^{2}$ \\ ${ }^{1}$ Geisinger Health System, 100 N Academy Ave, Danville, PA 17822, United States, ${ }^{2}$ Regional Hospital of Scranton, 746 \\ Jefferson Ave Scranton, PA 18510, United States.
}

\section{ABSTRACT}

Bouveret's syndrome is an unusual cause of gastric outlet obstruction secondary to gallstone impaction. It is so rare that it is commonly missed in clinical practice if it is not considered in differential diagnoses of intestinal obstruction more specifically in man who does not report the history of gall stone. Furthermore, there are no definitive guidelines on approach and management of this condition. We present a seventy eight year old man who developed acute abdominal symptoms due to impaction of a gallstone in the duodenum. He did not have history of gall stone. The diagnosis was supported via CT abdomen and Endoscopy. The patient was successfully managed by exploratory laparotomy followed by anterior pylorotomy to remove the stone without cholecystectomy and fistula repair.

Keywords: Bouveret's syndrome; cholelithiasis; enterolithotomy; gallstone ileus.

\section{INTRODUCTION}

Bouveret's syndrome is a rare condition of duodenal or gastric outlet obstruction secondary to an impacted gallstone in the duodenum or pylorus in association with a cholecystoduodenal fistula..$^{1,2}$ The syndrome is named after Leon Bouveret, the renowned French authority on gastric diseases. He reported first two cases of gastric outlet obstruction due to gallstones in $1896 .^{3,4}$ This rare condition tends to occur more commonly in women $(65 \%)$ with a median age of 74.1 years in the setting of history of gall stone. ${ }^{5}$ Our case is even more unusual because of its occurrence in man without prior report of cholelithiasis.

\section{CASE REPORT}

A seventy eight year old Caucasian man with a history of dementia presented to the emergency department with several episodes of projectile vomiting and severe abdominal pain for past two to three hours. The pain was localized to epigastrium. It was non-radiating and continuously progressing. Patient reported that he never had gastrointestinal symptoms in the past. On exam, he appeared ill and was dehydrated. His temperature was 101 degrees Fahrenheit and pulse was 113 per minute. His abdominal exam was significant for epigastric tenderness without peritoneal signs. He had leukocytosis of 14,000 per microliter. Rest of his labs including a liver function test was unremarkable. Abdominal X-ray demonstrated few non-specific distended loops of small bowel in the upper abdomen without free intraabdominal air. A CT scan with oral contrast showed a focal filling defect in proximal small bowel without flow of contrast beyond that point. There was no air in the biliary tree. He was ordered for a nothing per mouth. He received intravenous fluid hydration along with intravenous ciprofloxacin and metronidazole. An upper endoscopy was performed which revealed a darkcolored "sphere-like" object impacted in the lumen of the descending part of duodenum. It was immediately recognized by the endoscopist to be a gallstone (Figure

Correspondence: Dr Mukul Bhattarai, Geisinger Health System, $100 \mathrm{~N}$ Academy Ave, Danville, PA 17822, United States. Email: drmukulbhattarai@gmail.com. 
1, 2). However, it was so impacted that endoscopic retrieval was not possible. General surgery was consulted immediately and decision for surgery was made. Exploratory laparotomy was performed; thereby a three centimetre spherical gallstone was successfully removed from duodenum via anterior pylorotomy. Due to significant inflammation in right upper quadrant and absence of residual stones in the gall bladder, cholesytectomy and the closure of fistula was not performed. Patient did well after the surgery and was discharged on day six. He remained asymptomatic without any gastrointestinal symptoms even after six month follow up.

\section{DISCUSSION}

Gallstone ileus is an unusual complication of cholelithiasis, occurring in less than 0.5 percent of patients of cholelithiasis. ${ }^{6}$ Gallstone is responsible for approximately 1 to 4 percent of all cases of mechanical small bowel obstruction where it mostly gets lodged in ileum $(86 \%)$, and Bouveret's syndrome only represents $1-3 \%$ of Gallstone ileus. ${ }^{1,6-8}$ Earlier, Bouveret's syndrome was associated with high mortality rate up to $30 \%$. However, with the implementation of modern technologies, the mortality has been reduced but is still as high as $12 \%$ in recent years which is mainly due to its association with elderly patients who already have multiple comorbidities and also partly due to delayed diagnosis. ${ }^{1,2}$ Therefore, it is important to recognize the presenting symptoms of this rare entity which are epigastric pain, nausea, and vomiting in the presence of notable risk factors such as history of gallstones measuring 2-8 cm, long history of biliary disease, repeated episodes of acute cholecystitis, female sex and advanced age (over 60 years). ${ }^{1,3}$ But the absence of a preceding history of gallstone disease in a male patient does not exclude this entity such as in our case.

The diagnosis and management of this condition is very challenging despite availability modern technologies. Classically, Rigler's triad (signs of intestinal obstruction, ectopic gallstone, and pneumobilia) on plain abdominal $\mathrm{X}$-ray is used to diagnose gallstone ileus. However, this triad is present on plain X-rays in less than $15 \%$ of cases and relatively common in upper gastrointestinal series. ${ }^{2,5}$ Similarly, abdominal CT (Computed Tomography) and US (Ultrasonography) are noninvasive tests commonly used to facilitate diagnosis of this condition. ${ }^{5}$ However, multidetector computeriszed tomography (CT) and contrast-enhanced magnetic resonance cholangiopancreatography (CE-MRCP) may offer better visualization of the impacted stone. ${ }^{1}$ One of the comprehensive reviews of this condition has shown that EGD (esophagogastroduodenoscopy) is not only considered diagnostic procedure but also is therapeutic, thereby avoiding the high risk of surgical management. ${ }^{5}$

Currently, no definitive guidelines or standards have been proposed to manage this condition. However, enterotomy or gastrotomy with or without cholecystectomy and fistula repair is the most common surgical method applied in reported cases in literature..$^{2,5}$ Studies have shown that fistula repair may be unnecessary such as in our case as these fistulas may close spontaneously especially if the cystic duct is patent and residual gallstones are not present. ${ }^{5,9}$ In our case, gallstone located in duodenum was successfully removed via anterior pylorotomy. The cholecystectomy and closure of the fistula were not performed because there was an increased risk of injury to other structures due to the presence of significant inflammation in right upper quadrant. In addition, there was no residual gallstone present in gall bladder.
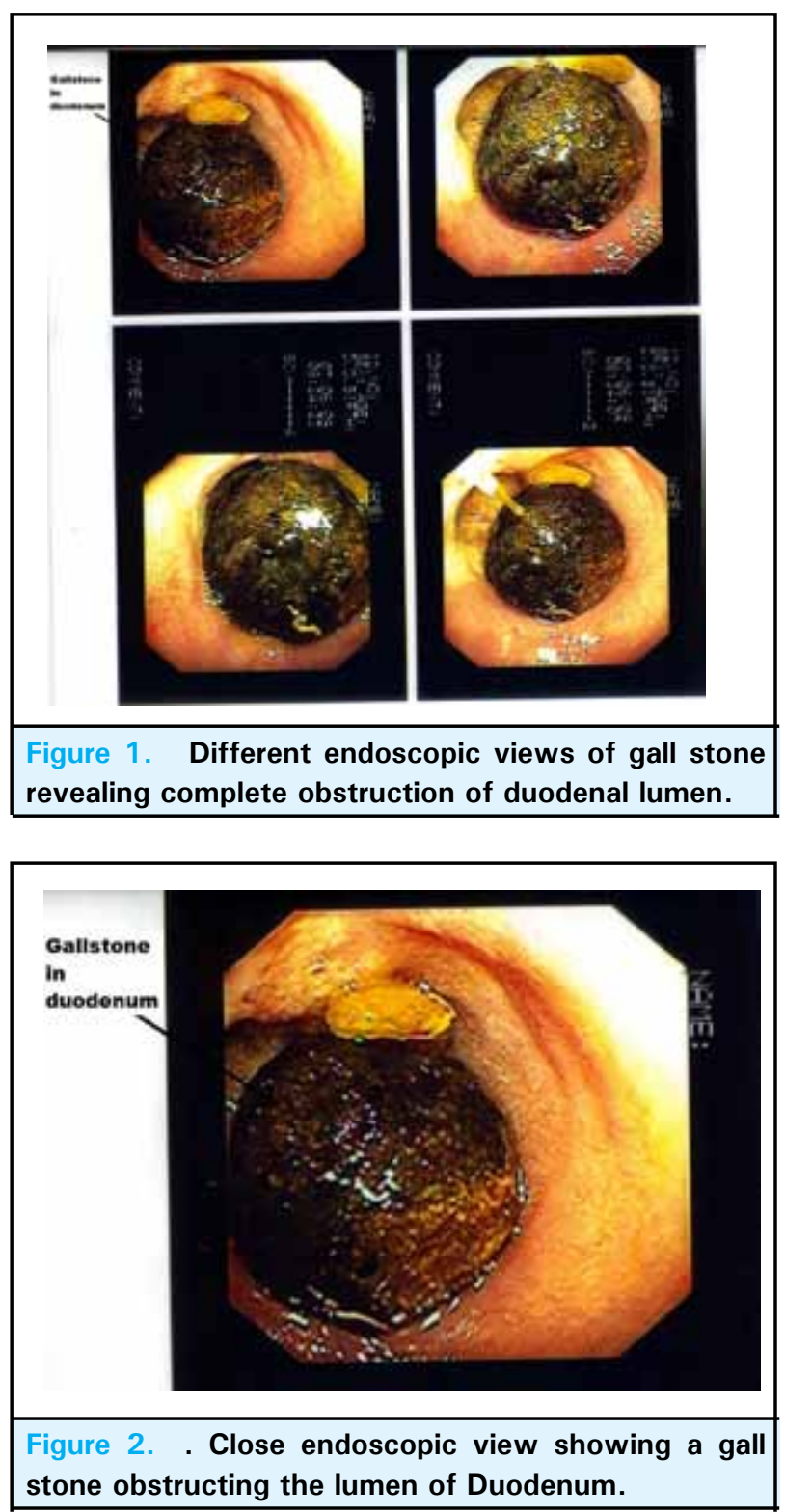
Besides surgery, there are also several reports of endoscopic management discussed in the literature but success rates have been minimal due to difficulties in removing impacted gallstones such as in our case. ${ }^{4,5,9}$ Still, endoscopic retrieval or other less invasive technique such as lithotripsy has been considered as an useful alternatives to surgery when there is high risk of perioperative complications. ${ }^{2,5}$ The decision to operate should be taken on an individual basis after evaluating patient's general condition, age, stone size, comorbidities influencing the operative risk, and expertise of surgical and endoscopic teams.
In a summary, Bouveret's syndrome should be included in the differential diagnosis during an assessment of any elderly patient including male who presents with the features of duodenal or gastric outlet obstruction even in the absence of history of gallstone disease. The management of Bouveret's syndrome should be individualized. Based on our case and literature review, we recommend abdominal imaging with CT scan if abdominal $\mathrm{X}$-ray is inclusive; also recommend evaluating endoscopically to retrieve the stone, if failed, should consider exploratory laparotomy which may not constitute fistula repair and cholecystectomy. Therefore, careful scrutiny of each case is very essential to manage this rare condition.

\section{REFERENCES}

1. Sunumu O. Bouveret syndrome: evaluation with multidetector computed tomography and contrast-enhanced magnetic resonance cholangiopancreatographym. Ulus Travma Acil Cerrahi Derg. 2013;19(4):375-379.

2. Nabais C, Salústio R, Morujão I, Sousa FV, Porto E, Cardoso C, et al. Gastric outlet obstruction in a patient with Bouveret's syndrome: a case report. BMC Res Notes. 2013;6:195.

3. Bouveret L. Sténose du pylore adhérent à la vésicule. Rev Med (Paris). 1896;16:1-16.

4. Doycheva I, Limaye A, Suman A, Forsmark CE, Sultan S. Bouveret's syndrome: case report and review of the literature. Gastroenterol Res Pract. 2009;2009:914951.

5. Cappell MS, Davis M. Characterization of Bouveret's Syndrome: A Comprehensive Review of 128 Cases Am J Gastroenterol. 2006;101(9):2139-46.
6. Reisner, RM, Cohen, JR. Gallstone ileus: A review of 1001 reported cases. Am Surg. 1994;60:441.

7. Langhorst J, Schumacher B, Deselaers T, Neuhaus H. Successful endoscopic therapy of a gastric outlet obstruction due to a gallstone with intracorporeal laser lithotripsy: A case of Bouveret's syndrome. Gastrointest Endosc. 2000;51:209-13.

8. Ferreira, LE, Topazian, MD, Baron, TH. Bouveret's syndrome: diagnosis and endoscopic treatment. Clin Gastroenterol Hepatol. 2008;6:e15.

9. Kishi K, Yamada K, Sugiyama T. Gastric Outlet Obstruction Caused by a Large Gallstone in the Duodenum (Bouveret's Syndrome). Clin Gastroenterol Hepatol. 2008;6(3):e11. 